\title{
El art. 73 del Convenio Colectivo de Trabajo de Docentes Universitarios. La estabilidad como premisa
}

\author{
The article 73 of the Collective Bargaining \\ Agreement for University Teachers. Stability \\ as a premise \\ $O$ artigo 73 do Acordo Coletivo de Trabalbo de \\ Professores Universitários. A estabilidade como \\ premissa
}

L'article 73 de la conventione collective du travail des enseignants universitaires

艺术《大学教师集体工作协议》第73条。稳定性为前提

Alejandro Emilio Berardi ${ }^{1} \mid \begin{aligned} & \text { Universidad Nacional, } \\ & \text { de Mar del Plata, Argentina }\end{aligned}$

Revista Derechos en Acción ISSN 2525-1678/ e-ISSN 2525-1686

Año 4/Nº 13, Primavera 2019 (21 septiembre a 20 diciembre), 190-201

DOl: https://doi.org/10.24215/25251678e336

ORCID: https://orcid.org/0000-0002-3968-963X

Recibido: 07/02/2018

Aprobado: 25/09/2019

Resumen: La propuesta de este trabajo consiste en realizar un análisis de la cláusula transitoria agregada como art. 73 en el Convenio Colectivo de Trabajo de los Docentes Universitarios -en adelante CCT-, a partir de la experiencia vivida en la Universidad Nacional de Mar del Plata (UNMdP), en donde luego de casi dos años de discusión en el marco de la paritaria de nivel particular se suscribió un acuerdo estableciendo diversos mecanismos para el ingreso de los docentes interinos comprendidos en la citada la citada cláusula. En el entendimiento de

\footnotetext{
1 Abogado, Asesor Legal de la Agremiación Docente Universitaria Marplatense -ADUM-, Email: berardialejandro@hotmail.com.
} 
que el CCT constituye una formidable oportunidad para avanzar en el reconocimiento de los derechos laborales de los trabajadores docentes universitarios, el referido art. 73 puede considerarse un verdadero pilar. En efecto, el primer derecho, básico, elemental, que debemos exigir y proteger, es el derecho a la estabilidad. Esta norma propicia el ingreso a la carrera docente de miles de trabajadores que hoy se desempeñan en forma precaria, con designaciones interinas, que requieren periódicas renovaciones y sujetos en muchos casos a innumerables presiones.

Palabras claves: Convenio Colectivo-Trabajador Docente- UniversidadEstabilidad

Abstract: The proposal of this work consists of carrying out an analysis of the transitory clause added as art. 73 in the Collective Labor Agreement of University Teachers - hereinafter CCT - from the experience lived in the National University of Mar del Plata (UNMdP) where, after almost two years of discussion in the framework of the parity of particular level an agreement was signed establishing diverse mechanisms for the entrance of the interim teachers included in the mentioned clause. In the understanding that the CCT constitutes a formidable opportunity to advance in the recognition of the labour rights of university teaching workers, the aforementioned article 73 can be considered a real pillar. Indeed, the first, basic, elementary right that we must demand and protect is the right to stability. This norm favors the entry into the teaching career of thousands of workers who today work in a precarious manner, with interim appointments that require periodic renewals and subject in many cases to innumerable pressures.

Keywords: Collective Bargaining Agreement- Teacher Worker- University- Stability

Resumo: A proposta deste trabalho consiste em realizar uma análise da cláusula transitória acrescentada como artigo 73 no Acordo Coletivo de Trabalho dos Docentes Universitários, com base na experiência vivida na Universidade Nacional de Mar del Plata (UNMdP), onde após de quase dois anos de discussão no âmbito da negociação de nível individual foi assinado um acordo estabelecendo vários mecanismos para a admissão de professores interinos incluídos na cláusula mencionada. No entendimento de que o Acuerdo Coletivo constitui uma oportunidade formidável para avançar no reconhecimento dos direitos 
trabalhistas dos docentes universitários, 0 artigo 73 pode ser considerado um verdadeiro pilar. Em efeito, o primeiro direito, básico, elemental, que devemos exigir e proteger, é o direito à estabilidade. Essa regra incentiva a entrada na carreira docente de milhares de trabalhadores que hoje trabalham precariamente, com designações interinas, que exigem renovações periódicas e sujeitas em muitos casos a inúmeras pressões.

Palavras-chave: Acordo Coletivo-Trabalhador docente UniversidadeEstabilidade

Résumé: Ce travail propose d'effectuer une analyse de la clause transitoire ajoutée comme article 73 de la convention collective des professeurs d'université -ci-après dénommée CCT-. Cela, à partir de I'expérience vécut à l'université nationale de Mar del Plata (UN Mdp) où, après presque deux ans de discussions salariales au niveau individuel, on a signé un accord qui dispose des divers mécanismes d'admission des enseignants par intérim dans la clause susmentionnée. Considérant que le CCT constitue une formidable opportunité de faire progresser la reconnaissance des droits du travail des travailleurs universitaires, I'article 73 peut être considéré comme un véritable pilier. En effet, le premier droit, fondamental, élémentaire, que nous devons exiger et protéger, est le droit à la stabilité. Cette règle favorise l'entrée dans la carrière d'enseignement de milliers de travailleurs qui travaillent aujourd'hui de manière précaire, avec des désignations par intérim, qui ont besoin des renouvellements périodiques et qui, dans de nombreux cas, sont soumis à d'innombrables pressions.

Mot-clés: conventione collective, travailleur enseignant, Université, estabilité

\section{Introducción}

El art. 73 del CCT establece: Las Instituciones Universitarias Nacionales, a través de la Comisión Negociadora de Nivel Particular, dispondrán los mecanismos para la incorporación a carrera docente de los docentes que revistan como interinos, y que a la firma del presente convenio tengan cinco años o 
más de antigüedad en tal condición, en vacantes definitivas de planta estable".

La norma merece dos consideraciones iniciales. En primer lugar, impone una obligación taxativa a las Instituciones Universitarias de incorporar a los docentes interinos con más de cinco años de antigüedad, que no es ni más ni menos que poner fin a una manifiesta irregularidad. En efecto, la continuidad en un cargo en forma interina por tanto tiempo excede toda razonabilidad, pero además, constituye una ilegalidad, ya que la mayoría de los estatutos de las diferentes instituciones universitarias, establecen la obligación de llamar a concurso luego de determinado periodo. En el caso de la UNMdP, estatuto fue modificado recientemente, estableciendo la obligación de incluir el llamado a concurso del cargo en la ordenanza de designación de un docente interino en un cargo de creación o que ha quedado vacante. Pero en la redacción anterior, el estatuto establecía que la designación interina se efectuaba hasta tanto se realice el concurso, que debía ser convocado dentro del año.

En segundo lugar, en parte por lo antedicho, la incorporación no puede llevarse a cabo mediante concurso público y abierto de antecedentes y oposición (tal como establece el art. 11 para el acceso a la carrera docente), sino mediante otros mecanismos que serán acordados en cada Universidad mediante discusión paritaria. Justamente por tratarse de situaciones especiales, ameritan soluciones diferentes. Por si quedara alguna duda, el mismo art. 73 establece en su tercer párrafo: "Para el caso de los docentes que revistan como interinos, y que a la firma del presente convenio tengan entre dos a cinco años de antigüedad en tal condición, en vacantes definitivas de la planta estable, las Instituciones Universitarias Nacionales deberán cumplir con el procedimiento establecido en el art. 11 del presente Convenio". Vemos entonces que en este caso sí debe llamarse a concurso público y abierto de antecedentes y oposición, diferenciándose claramente del primer párrafo, que refiere, como se dijo, a otros "mecanismos". 
No debe soslayarse la enorme importancia de estas dos disposiciones que promueven el ingreso a la carrera docente de todos los interinos con más de dos años de antigüedad. Se ratifica que es éste un derecho esencial de todos los trabajadores docentes universitarios. Tanto el derecho a la carrera docente como su corolario, la estabilidad, que debe ser absoluta, con la única excepción de los mecanismos de reválida previstos en el art. 12, que si bien son reglamentados por cada Universidad, deben respetar las claras limitaciones que impone dicho artículo.

El segundo párrafo del art. 73 no es menos importante, establece: "Hasta tanto se resuelva la situación de los mismos, no se podrá modificar en detrimento del docente, la situación de revista y/o condiciones de trabajo, por acción y omisión ...". Constituye una ratificación de que los docentes interinos comprendidos en la primera parte de la norma, han adquirido ya un derecho a cierto grado de estabilidad en su cargo, situación que solo podrá ser modificada una vez que se hayan desarrollado las instancias que se establezcan para la regularización. Pero además, de cierta forma, esta disposición suple la carencia de plazos específicos para el cumplimiento de estas pautas. De manera que los perjuicios que puedan generar las eventuales dilaciones en el cumplimiento por parte de las distintas Universidades de lo allí establecido, se vean morigerados, otorgándole al docente interino, mediante esta garantía, la tranquilidad de que permanecerá en el cargo durante el periodo de discusión en la paritaria para definir los mecanismos de ingreso, pero también durante el desarrollo de los diferentes procesos que se llevarán a cabo.

Idéntica garantía establece el tercer párrafo del art. 73, ya que luego de disponer la obligación de llamado a concurso para docentes interinos con dos años de antigüedad, reitera: "No se podrá modificar en detrimento del docente, la situación de revista y/o condiciones de trabajo, por acción y omisión hasta la cobertura del cargo por concurso público y abierto de antecedentes y oposición". 


\section{La paritaria de Nivel Particular en la UNMdP}

La norma convencional que venimos analizando permitió en la Universidad Nacional de Mar del Plata -UNMdP- el inicio de una etapa de debate que resultó sumamente interesante y que trascendió la discusión puntal de la puesta en vigencia, en forma concreta, del mentado art. 73.

Como ya se dijo, se llevó a cabo un proceso de discusión en el ámbito de la paritaria de nivel particular que llevó casi dos años y que concluyó con un acuerdo que fue suscripto en la sede local del Ministerio de Trabajo, Empleo y Seguridad Social de la Nación. El ámbito elegido tuvo un fin más simbólico que jurídico. Se trataba de enmarcar claramente la cuestión, que no quedara ninguna duda de que se estaba suscribiendo un convenio en el que el sector empleador se comprometía a llevar a cabo determinadas acciones que garantizaban determinados derechos de los trabajadores docentes universitarios.

No es un tema menor. Quienes participamos de la actividad sindical en el ámbito universitario, no podemos ignorar una realidad palpable cotidianamente, la enorme reticencia del docente a asumir su condición de trabajador. Los motivos son variados y exceden con creces este trabajo, pero es indudable que debe tenerse en cuenta este hecho. Por ello es tan importante que abandonemos los eufemismos, que muchas veces utilizamos para evitar herir susceptibilidades o pensando que evitamos conflictos. En más de una oportunidad se han suscripto acuerdos paritorios que consistían en compromisos de las partes de presentar determinada propuesta de ordenanza en forma conjunta. De modo tal que la cuestión se zanjaba o el reclamo se satisfacía en la negociación, pero no adquiría valor legal hasta tanto no se dictara la ordenanza. No es sino una manera de quitar valor a la negociación colectiva por parte de las autoridades universitarias, que se reservan así la exclusividad en la "creación del derecho". Sin embargo es difícil que los trabajadores docentes asuman la importancia de exigir el reconocimiento del acuerdo paritario como norma legal, obligatoria para las 
partes y terceros. Que comprendan cabalmente que las condiciones de trabajo deben ser discutidas en el ámbito de la negociación paritaria. Que los derechos reconocidos en una convenio no pueden modificarse sino mediante otro convenio, es decir con la participación de los trabajadores, en tanto que la modificación de una ordenanza, depende exclusivamente de la mayoría circunstancial en el respectivo consejo, sea superior o académico.

Y nos enfrentamos también a otra dificultad. Los docentes que ejercen cargos de gestión y que en consecuencia durante el ejercicio de sus funciones constituyen la "parte empleadora", no asuman claramente dicho carácter. Los roles muchas veces se confunden y las discusiones se complican más de lo necesario.

Como dijimos los debates fueron muy ricos, ya que no se limitaron a las posturas entre las dos partes que conforman la paritaria, sino también entre los propios trabajadores. De hecho, durante el proceso de discusión paritaria, además de numerosas reuniones en las que participaron los docentes comprendidos en la norma convencional, se llevaron a cabo tres asambleas, en las que se fueron otorgando diferentes mandatos a los representantes paritarios. El acta solamente se firmó una vez que una asamblea de afiliados aprobó, luego de un extenso debate, el pre acuerdo al que se había arribado.

Fue necesario además superar algunos prejuicios. La idea de que el ingreso, aún de docentes que llevaban muchísimos años desempeñándose en determinado cargo, por mecanismos diferentes al concurso abierto de oposición y antecedentes, atentaba contra la "excelencia académica". Es más, se corría el riesgo de establecer la dicotomía "derechos laborales de los docentes" versus "excelencia académica". El riesgo era entender la discusión como la búsqueda de consensos para "compatibilizar" estos supuestos antagonismos con el menor daño posible. Colocar a las autoridades de la Universidad como aquellos que debían resguardar "la excelencia académica" frente al avance sindical que pretendía hacer ingresar "por la ventana" a la planta regular 
a los docentes interinos. Saldar esta cuestión fue esencial, el debate permitió poner luz sobre el tema y demostrar que no existe tal antagonismo, sino que, por el contrario, el camino del respeto de los derechos laborales de los docentes es la mejor forma de alcanzar mayores niveles de excelencia. Pero además, que no solamente es falsa la dicotomía, sino que nunca puede plantearse, ni siquiera pensarse, que determinados objetivos, sean cuales fueran, pueden alcanzarse violentando derechos $\mathrm{y}$ precarizando el trabajo docente.

Ahora bien, puestos a analizar cuáles podían ser los mecanismos más adecuados para que los docentes comprendidos en la norma convencional ingresaran a carrera docente, se partió de un acuerdo básico a partir del cual comenzar a discutir, que incluyó cuatro aspectos considerados como punto de partida.

En primer lugar que era necesario buscar alguna diferenciación en razón de la antigüedad, no parecía adecuado que se tratara de igual manera a todos los interinos. Tengamos en cuenta que en la UNMdP el universo de trabajadores comprendidos es muy variado, desde aquellos que tienen el mínimo exigido de cinco años hasta quienes ya llevan veinte o más años de antigüedad como interinos en su cargo.

En segundo lugar, que esos docentes que tienen muchísimos años de antigüedad en el mismo cargo, designados anualmente, sujetos a distintas formas de evaluación, en fin, que habían acreditado sobradamente su idoneidad debía ingresar sin necesidad de mecanismo evaluativo alguno. La cuestión se limitaba a

Se llegó también a un consenso básico respecto a los restantes docentes, todos deberían pasar por algún mecanismo de evaluación.

Por último, debían atenderse los casos de quienes, además de superar los cinco años de antigüedad, estaban cercanos a cumplir los requisitos necesarios para obtener el beneficio jubilatorio.

En ese entendimiento se acordó un corte relacionado con la antigüedad en el cargo. Se establecieron entonces cuatro 
grupos. Un primer grupo integrado por quienes poseían menos de cinco años de antigüedad, comprendidos en el párrafo tercero de la art. 73, sobre cuyos cargos se estableció la obligación de llamados a concurso público y abierto, de oposición y antecedentes. Sin bien el art. 73 del CCT ya establece taxativamente esta obligación, la importancia de incluir a este grupo radique ens que, como se explicará más adelante, el acuerdo incluyó diferentes plazos para la puesta en marcha y desarrollo de los distintos procedimiento. Se garantiza entonces que no se dilate el llamado a concurso de todos estos cargos.

Para aquellos cargos que cuentan con designación de interinos con más de cinco años de antigüedad y menos de diez, se llevará a cabo un concurso público de oposición y antecedentes circunscripto al área o, de no existir división por áreas en la unidad académica se circunscribe a los integrantes del departamento. Pero además se limita la inscripción a aquellos docentes que hayan sido designados con anterioridad al 30 de junio de 2010, es decir que también posean una antigüedad mayor a cinco años. Por último se establece que se reconocerá al aspirante que detente el cargo interino objeto del concurso una valoración adicional obligatoria en el puntaje final que consistirá en veinte puntos porcentuales por encima de la valoración básica total. Hay claramente una intención de brindar mayores posibilidades a los interinos que poseen el cargo, de manera de insistir en la idea de que se trata de mecanismos para que el interino pueda acceder a la regularización de su cargo siempre que reúna las condiciones y no una búsqueda del docente "más calificado" como sería un concurso totalmente abierto.

Un tercer grupo es el de aquellos cargos interinos con designaciones anteriores al 30 de junio de 2005, esto es, docentes interinos con una antigüedad mayor a diez años. Aquí el concurso es cerrado al cargo. La intención en este caso es que, aquél docente que ha ejercido el cargo por más de diez años no tenga que someterse a una "competencia", sino que solamente deba ratificar en un procedimiento concursal que posee la idoneidad suficiente para continuar en el cargo. 
En el cuarto grupo se incluye a los interinos con más de quince años de antigüedad en el cargo. En este caso se establece un "concurso cerrado de ratificación de antecedentes" en el que solamente deberán presentar ante una comisión creada ad hoc documentación que acredite el cumplimiento de las condiciones de ingreso establecidas en la ordenanza respectiva y haber obtenido aprobación el Plan de Trabajo docente correspondiente al ciclo lectivo en curso, además de presentar su currículum de carrera docente. Se advierte que se trata de verificar exclusivamente cuestiones formales, de ninguna manera consiste en alguna clase de evaluación ya que, como se dijo anteriormente, se entiende que un trabajador que durante más de quince años ha cumplido tareas docentes en el cargo, habiendo sido designado anualmente, ha demostrado acabadamente su idoneidad. Además de tratarse de una mayúscula irregularidad la omisión en el llamado a concurso durante tanto tiempo con el consiguiente perjuicio al docente al que se le privó de estabilidad y de desarrollar su carrera. Para garantizar que no se desnaturalice el denominado "concurso de ratificación de antecedentes", se establece que un veedor gremial integrará la comisión ad hoc y participará en la elaboración del dictamen.

Como se dijo anteriormente, se contempló también la situación de aquellos docentes próximos a jubilarse. El acuerdo establece que quienes al momento de la publicación de CCT estaban a cinco años o menos de la edad para acogerse al beneficio jubilatorio pueden mantener su situación de revista en los cargos involucrados. De manera que, salvo que opte por alguno de los mecanismos establecidos, el docente puede continuar hasta al momento de su jubilación, con la garantía de la estabilidad en su cargo, aún interino, sin necesidad de someterse a procedimiento de evaluación alguna.

Solo cabe aclarar que el acuerdo incluye también una serie de disquisiciones respecto a distintas situaciones que pudieran darse en cuanto a formas contar la antigüedad, casos de duplicidad de cargos, en fin, intenta abarcar la mayor cantidad de 
casos posibles. Asimismo, que se establecieron plazos para cada etapa de los distintos procedimientos y las posibles acciones a seguirse en caso de incumplimiento.

Debe tenerse en cuenta que cada unidad académica tiene su propia realidad, su lógica de funcionamiento y esto hace más complejo intentar que todo avance al mismo ritmo y que en todos los casos se verifique idéntico cumplimiento. Pero lo fundamental es que el proceso ya se ha iniciado, y significará que más de ochocientos cargos interinos, que constituye alrededor de un $17 \%$ de la totalidad de cargos existentes en la UNMdP, pasarán ser regulares. Y que aproximadamente seiscientos docentes, de aproximada tres mil quinientos, que constituye la planta total de docentes que hoy se desempeñan en la UNMdP ingresarán a la carrera.

\section{Consideraciones finales}

Luego de un arduo trabajo de muchos años, representantes de casi todas las universidades públicas nacionales y de todos los gremios del sector, suscribieron el primer convenio colectivo de trabajo de los docentes universitarios. Indudablemente un hecho histórico. Pero que haya sido el colectivo de trabajadores docentes universitarios uno de los últimos en tener su convenio colectivo no es una casualidad. Debieron vencerse muchas resistencias y otras aún persisten y dificultan su puesta en marcha, su aplicación concreta.

Todavía algunos ven a la negociación colectiva en general y a este convenio en particular como un avance contra la autonomía universitaria. Hay quienes sostienen que la estabilidad atenta contra la excelencia académica. En algunas Universidades se sostiene aún la periodicidad de los cargos. Es más, en alguna asamblea universitaria se presentaron mociones que apuntaban a desconocer la vigencia del CCT.

Por ello, la puesta en vigencia del convenio es una lucha cotidiana y el acuerdo logrado en la UNMdP, como también 
ha ocurrido en otras universidades, para poner en marcha uno de los artículos que más polémicas ha desatado, constituye un hito y el inicio de un largo recorrido que debe continuarse.

La defensa irrestricta del derecho a negociar colectivamente las condiciones de trabajo debe ser una bandera inclaudicable de todos los trabajadores docentes universitarios. 